\title{
A Prospective Multicenter Study on Fever of Unknown Origin
}

\author{
The Yield of a Structured Diagnostic Protocol
}

\author{
Chantal P. Bleeker-Rovers, MD, Fidel J. Vos, MD, Elisabeth M. H. A. de Kleijn, MD, PhD, \\ Aart H. Mudde, MD, PhD, Ton S. M. Dofferhoff, MD, PhD, Clemens Richter, MD, PhD, \\ Tineke J. Smilde, MD, PhD, Paul F. M. Krabbe, PhD, Wim J. G. Oyen, MD, PhD, \\ and Jos W. M. van der Meer, MD, PhD
}

\begin{abstract}
We conducted a prospective study to update our knowledge of fever of unknown origin (FUO) and to explore the utility of a structured diagnostic protocol. From December 2003 to July 2005, 73 patients with FUO were recruited from 1 university hospital $(n=40)$ and 5 community hospitals $(n=33)$ in the same region in The Netherlands. FUO was defined as a febrile illness of $>3$ weeks' duration, a temperature of $>38.3{ }^{\circ} \mathrm{C}$ on several occasions, without a diagnosis after standardized history-taking, physical examination, and certain obligatory investigations. Immunocompromised patients were excluded. A structured diagnostic protocol was used. Patients from the university hospital were characterized by more secondary referrals and a higher percentage of periodic fever than those referred to community hospitals. Infection was the cause in $16 \%$, a neoplasm in $7 \%$, noninfectious inflammatory diseases in $22 \%$, miscellaneous causes in $4 \%$, and in $51 \%$, the cause of fever was not found (no differences between university and community hospitals). There were no differences regarding the number and type of investigations between university and community hospitals. Significant predictors for reaching a diagnosis included continuous fever; fever present for $<180$ days; elevated erythrocyte sedimentation rate, C-reactive protein, or lactate dehydrogenase; leukopenia; thrombocytosis; abnormal chest computed tomography (CT); and abnormal ${ }^{18} \mathrm{~F}$-fluorodeoxyglucose
\end{abstract}

From Department of Internal Medicine (CPBR, FJV, JWMvdM), Department of Nuclear Medicine (CPBR, WJGO), and Department of Medical Technology Assessment (PFMK), Radboud University Nijmegen Medical Centre, Nijmegen; Nijmegen University Centre for Infectious Diseases (CPBR, FJV, WJGO, JWMvdM), Nijmegen; Division of Medical Oncology (EMHAdK), Department of Internal Medicine, University Medical Centre Nijmegen; Department of Internal Medicine (AHM), Slingeland Hospital, Doetinchem; Department of Internal Medicine (TSMD), Canisius-Wilhelmina Hospital, Nijmegen; Department of Internal Medicine (CR), Rijnstate Hospital, Arnhem; and Department of Internal Medicine (TJS), Jeroen Bosch Hospital, 's-Hertogenbosch; The Netherlands.

This study was funded with internal resources. The funding source had no involvement in study design, in the collection, analysis, and interpretation of data, in the writing of the report or in the decision to submit the paper for publication.

Address reprint requests to: Chantal Bleeker-Rovers, Department of Internal Medicine, Radboud University Nijmegen Medical Centre, P.O. Box 9101, 6500 HB Nijmegen, The Netherlands. Fax: 3124 3541734; e-mail: c.bleeker-rovers@AIG.umcn.nl.

Copyright (C) 2007 by Lippincott Williams \& Wilkins

ISSN: 0025-7974/07/8601-0026

DOI: $10.1097 / \mathrm{md} .0 \mathrm{~b} 013 \mathrm{e} 31802 \mathrm{fe} 858$ positron emission tomography (FDG-PET). For future FUO studies, inclusion of outpatients and the use of a set of obligated investigations instead of a time-related criterion are recommended. Except for tests from the obligatory part of our protocol and cryoglobulins in an early stage, followed by FDG-PET, and in a later stage by abdominal and chest CT, temporal artery biopsy in patients aged 55 years or older, and possibly bone marrow biopsy, other tests should not be used as screening investigations.

(Medicine 2007;86:26-38)

Abbreviations: $\mathrm{ANCA}=$ antineutrophil cytoplasmic antibodies, $\mathrm{CAPD}=$ continuous ambulatory peritoneal dialysis, $\mathrm{CRP}=\mathrm{C}$-reactive protein, ESR $=$ erythrocyte sedimentation rate, FDG-PET $={ }^{18} \mathrm{~F}$-fluorodeoxyglucose positron emission tomography, FUO = fever of unknown origin, $\mathrm{HIV}=$ human immunodeficiency virus, $\mathrm{LDH}=$ lactate dehydrogenase, $\mathrm{NHL}=$ non-Hodgkin lymphoma, NIID = noninfectious inflammatory diseases, NSAID $=$ nonsteroidal antiinflammatory drug, PDCs = potentially diagnostic clues, RUNMC $=$ Radboud University Nijmegen Medical Centre, SLE = systemic lupus erythematosus.

\section{INTRODUCTION}

espite recent advances in diagnostic techniques, fever of unknown origin (FUO) remains a formidable challenge. The 1961 definition $^{31}$ of FUO as an illness of more than 3 weeks' duration, fever $>38.3{ }^{\circ} \mathrm{C}\left(101{ }^{\circ} \mathrm{F}\right)$ on at least 2 occasions, and diagnosis uncertain after 1 week of hospitalization has been modified by removing the requirement that the evaluation must take place in the hospital ${ }^{30}$. Also, immunocompromised patients have been excluded, because these patients require an entirely different approach $^{10,11,30}$. To reduce selection bias, it has been proposed to change the quantitative criterion (diagnosis uncertain after 1 week of study) to the qualitative requirement that certain investigations have to be performed ${ }^{10,22,30}$.

The differential diagnosis of FUO is the most extensive in medicine, and construction of algorithms covering all possible causes is difficult. In general, infectious diseases explain about one-third of cases of FUO, followed by neoplasms and noninfectious inflammatory diseases (NIID) ${ }^{1}$. In the vast literature on FUO, no controlled trials or metaanalyses are available. In recent series from Europe and the United States, the percentage of patients with unexplained 
FUO varied from $7 \%$ to $53 \%{ }^{2,7,10,12,19,25,33-35}$. This variation is partly due to geographical factors and the different definitions of FUO used. Few studies have used uniform epidemiologic entry criteria or a structured diagnostic protocol; this may cause unintended selection bias and differences in diagnostic workup. Moreover, results of those studies cannot be easily extrapolated to an individual patient presenting with FUO.

We performed the current prospective study of patients with FUO recruited from 1 university hospital and 5 community hospitals in The Netherlands to update information on FUO using the revised definition of FUO. Since there are only 2 older studies that included patients from community hospitals ${ }^{15,19}$, the second aim was to compare the number and sort of additional diagnostic tests that were used and causes of fever between patients with FUO referred to a university hospital and patients referred to community hospitals. To minimize diversity in diagnostic management, a standardized diagnostic protocol was used; this was based on a previously performed in-depth inquiry into diagnostic management of FUO among Dutch internists ${ }^{8}$, on retrospective analysis of diagnostic management of patients with FUO in our university hospital ${ }^{3,7}$, and, most importantly, on the large prospective Dutch FUO studies of De Kleijn and colleagues ${ }^{9,10}$. Based on previous studies, showing that ${ }^{18} \mathrm{~F}$-fluorodeoxyglucose positron emission tomography (FDG-PET) contributed to the final diagnosis in $16 \%-69 \%$ of all patients with $\mathrm{FUO}^{3,4,6,20,26,27}$, FDG-PET was added to the protocol.

\section{PATIENTS AND METHODS}

\section{Hospitals}

From December 2003 to July 2005, patients with FUO were recruited from the Radboud University Nijmegen Medical Centre (RUNMC), a 950-bed university hospital and tertiary referral center for patients with classical FUO and periodic fever. Patients were also recruited from 5 community hospitals: Canisius Wilhelmina Hospital, Nijmegen (650 beds); Jeroen Bosch Hospital, 's-Hertogenbosch (450 beds); Rijnstate Hospital, Arnhem (750 beds); Slingeland Hospital, Doetinchem (450 beds); and Maxima Medical Centre, Veldhoven (500 beds). The study was approved by all local ethic committees.

\section{Patients}

FUO was defined as a febrile illness of $>3$ weeks' duration, a temperature $>38.3{ }^{\circ} \mathrm{C}$ on at least 2 occasions, without a diagnosis after standardized history-taking, standardized physical examination, and the following obligatory diagnostic investigations: erythrocyte sedimentation rate (ESR), C-reactive protein (CRP), hemoglobin, platelet count, leukocyte count and differentiation, electrolytes, creatinine, total protein, protein electrophoresis, alkaline phosphatase, aspartate aminotransferase, alanine aminotransferase, lactate dehydrogenase (LDH), creatine kinase, antinuclear antibodies, rheumatoid factor, urinalysis, at least
3 blood cultures, urine culture, chest X-ray, abdominal ultrasonography or computed tomography (CT), and tuberculin skin test. Periodic fever was defined as repeated episodes of fever with fever-free intervals of at least 2 weeks and apparent remission of the underlying disease. Immunocompromised patients, defined as patients with neutropenia (leukocyte count $<1.0 \times 10^{9} / \mathrm{L}$ and/or granulocyte count $\left.<0.5 \times 10^{9} / \mathrm{L}\right)$ during at least 1 week within the 3 months preceding the fever, known human immunodeficiency virus (HIV)-infection, known hypogammaglobulinemia (IgG $<50 \%$ of the normal value), or use of the equivalent of more than $10 \mathrm{mg}$ prednisone during at least 2 weeks in the previous 3 months, were excluded. All patients with FUO aged 18 years or older in the participating hospitals were eligible for inclusion. To minimize the chance of unintended selection bias, broad initial selection criteria were used. All records of nonimmunocompromised patients with fever on the internal medicine wards and outpatient clinics in all hospitals were reviewed for the criteria for FUO by either the first author or the local investigator. In a retrospective study of patients with FUO previously performed in our university hospital, it was found that blood cultures were performed in all patients with $\mathrm{FUO}^{7}$. Therefore, in the university hospital, records of all patients in whom blood cultures were performed were reviewed weekly for the criteria of FUO by the first author. All patients provided written informed consent.

\section{Diagnostic Workup}

In all patients primarily referred to 1 of the participating hospitals, a structured diagnostic protocol was used (Figure 1). In patients referred after investigations in another hospital, this diagnostic protocol was followed directly after referral. First, a complete and repeated history was taken, a complete physical examination was performed, followed by the obligatory investigations in a search for potentially diagnostic clues (PDCs). PDCs are defined as all localizing signs, symptoms, and abnormalities potentially pointing toward a diagnosis ${ }^{10}$. With the help of the PDCs identified from the history, examination, and tests, a limited list of probable diagnoses was made. Further diagnostic procedures were recommended to be guided by this list. Misleading PDCs are defined as PDCs not leading to the eventual diagnosis. In patients without PDCs or with only misleading PDCs, cryoglobulins were determined. When diagnosis was still uncertain, FDG-PET was performed. In patients with continuous fever with normal or false-positive FDG-PET results, bone marrow biopsy, temporal artery biopsy in patients older than 55 years, funduscopy, abdominal CT, and chest CT were performed. In patients with periodic fever, FDG-PET was performed during a symptomatic phase. In these patients, second-level diagnostic tests were recommended only when PDCs for infections, vasculitis syndromes, or malignancy were present, or when the clinical condition was deteriorating. In all patients with unexplained fever at this point, the recommended procedure was to repeat a thorough history and physical examination and to review laboratory results and imaging 


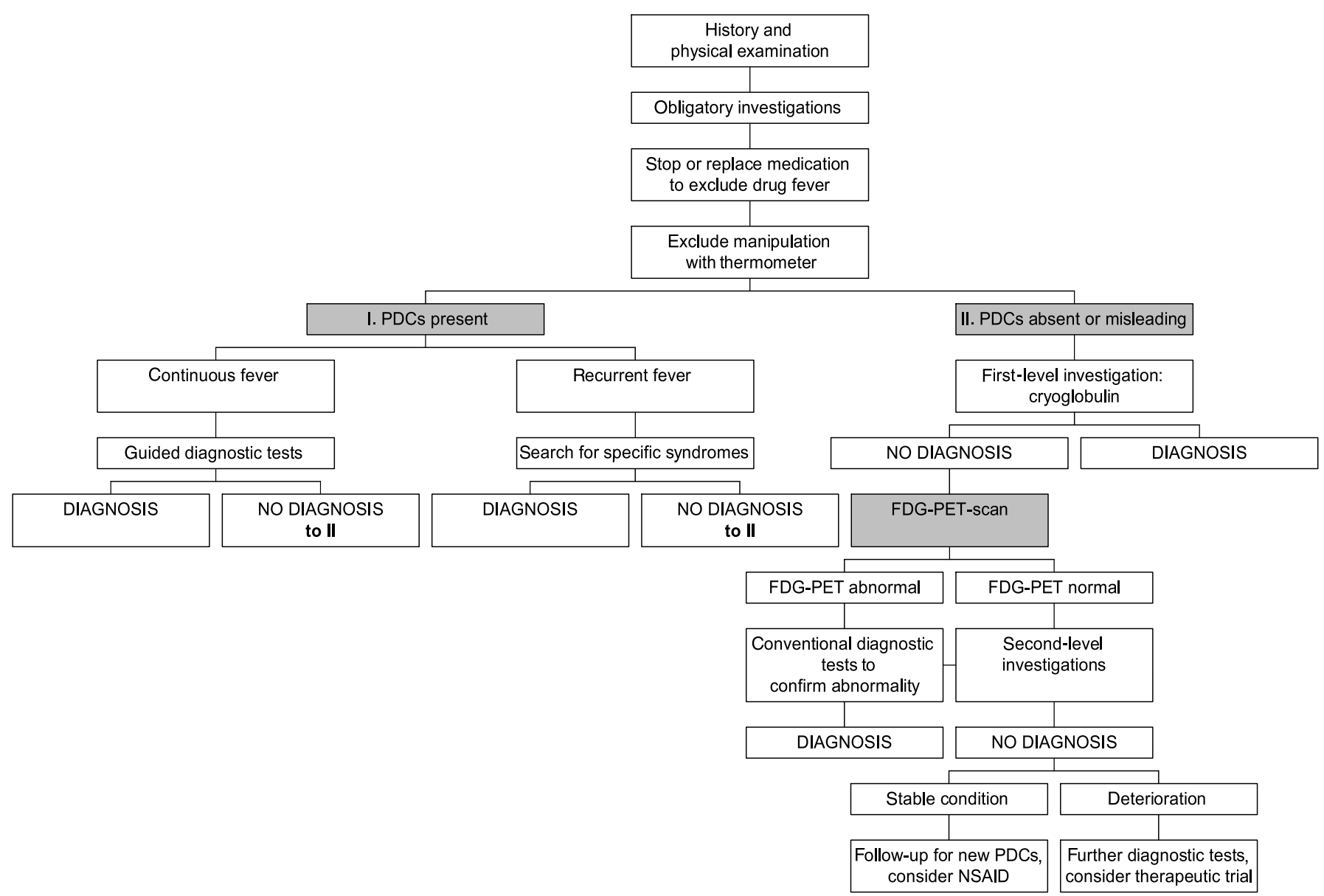

FIGURE 1. Diagnostic protocol that was used in all participating patients. Obligatory tests: erythrocyte sedimentation rate or C-reactive protein, hemoglobin, platelet count, leukocyte count and differentiation, electrolytes, creatinine, total protein, protein electrophoresis, alkaline phosphatase, ALAT, LDH, creatine kinase, antinuclear antibodies, rheumatoid factor, urinalysis, blood cultures $(n=3)$, urine culture, chest X-ray, abdominal ultrasonography (or CT) and tuberculin skin test. Second-level investigations: bone marrow biopsy, temporal artery biopsy in patients older than 55 years, funduscopy, abdominal CT, and chest CT.

studies including those from other hospitals. A further diagnostic workup or therapeutic trial with antibiotics, steroids, or antituberculous agents was recommended only in patients deteriorating without presenting new PDCs.

\section{Assessment of Diagnostic Results}

The results of diagnostic investigations were evaluated for their diagnostic contribution. Abnormal test results were categorized as "helpful in diagnosis" or "noncontributory to diagnosis." They were regarded as helpful in diagnosis or true positive when abnormal test results pointed to the cause of the fever. Abnormal results were regarded as noncontributory to diagnosis or false positive when the detected abnormality was considered to be unrelated to the illness causing the fever or when no final diagnosis could be made. Since in most cases a gold standard is not available, specificity and sensitivity could only be calculated assuming that negative results were true negative when further investigations or the final diagnosis did not contradict these results. Normal test results were categorized as true negative when no cause of the symptoms was identified despite an extensive diagnostic workup and clinical follow-up of at least 3 months. Normal test results were considered false negative when a disease was diagnosed that would usually cause positive test results. Most investigations were performed in each hospital according to local standards, because the scale of the study did not allow us to centralize these measurements and investigations.

\section{Diagnosis and Follow-Up}

The final diagnosis was established by the attending physician and the first author. A definite diagnosis was based on positive cultures, serology, histology, or internationally accepted criteria for certain diseases. When this was not possible, a probable diagnosis was made based on a combination of clinical follow-up, response to specific therapy and conventional imaging studies. Follow-up was performed by the first author by analysis of the patient's record, and in case no diagnosis was reached at the end of the study, by telephone calls to the attending physicians, and, in 
some cases, to the patients after a follow-up of at least 3 months and again after at least 6 months.

\section{Data Collection and Statistical Analysis}

Epidemiologic data; number, type, and results of all diagnostic tests performed; final diagnosis; treatment data; and follow-up data were registered for all patients in a structured database. Descriptive statistics for continuous variables are represented as means \pm standard deviations. Categorical variables are reported in terms of the number and percentage of patients affected. Differences between the patient groups were tested with unpaired Student t-tests for continuous variables and with Fisher exact tests for categorical variables. Differences were considered to be statistically significant at $p<0.05$. Sensitivity and specificity were calculated with $95 \%$ confidence intervals. All potential predicting factors for reaching a diagnosis (all parameters derived from the standardized history and physical examination, the obligatory diagnostic tests, and results of FDG-PET and abdominal and chest CT) were dichotomized. Patients with a final diagnosis were compared to patients without a final diagnosis using the Fisher exact test. Relative risks were calculated with $95 \%$ confidence intervals. A parameter was considered to be a significant predicting factor when the $95 \%$ confidence interval of the relative risk was $>1$.

\section{RESULTS}

\section{Clinical Features}

Between December 2003 and July 2005, 75 patients with FUO were identified by review of patient records or by direct report of treating physicians (Figure 2). No additional patients were identified by reviewing the records of all patients in whom blood cultures were performed during the study period. Two patients refused participation, so 73 patients were enrolled. The results of 73 patients $(33$ male and 40 female patients) with a mean age of 54 years (range, 26-87 yr) were evaluated: 40 patients were recruited from the university hospital (RUNMC) and 33 from the other hospitals (Table 1). Twenty-five patients (34\% of all patients, $63 \%$ of patients recruited from RUNMC) were referred to RUNMC after extensive investigation in another hospital. All patients recruited from the community hospitals were referred by a general practitioner $(\mathrm{p}<0.0001)$. Twentyfive patients $(34 \%)$ had periodic fever: 20 of these patients $(80 \%)$ were enrolled from RUNMC and 5 from community hospitals $(\mathrm{p}<0.005)$. The patient group from RUNMC was thus characterized by a higher percentage of secondary referrals and significantly more patients with periodic fever when compared to the patient group recruited from the community hospitals. Of all FUO patients, 85\% were hospitalized for a median duration of 24 days (range, 3-177 d). Significant differences between the university hospital and the community hospitals regarding the percentage of patients hospitalized or duration of hospitalization were absent.

\section{Diagnosis and Outcome}

An infectious disease was the cause of the fever in 12 patients $(16 \%)$, a neoplasm in 5 patients (7\%), NIID in 16 patients $(22 \%)$, and miscellaneous causes in 3 patients $(4 \%)$ (Table 2). The proportion of patients in each diagnostic category did not differ significantly between RUNMC and the community hospitals. NIID were diagnosed significantly more often in patients who were directly referred, compared to those referred for a second opinion (41\% vs. $9 \%, \mathrm{p}=$ 0.04). Diagnoses were definitely confirmed by biopsy,

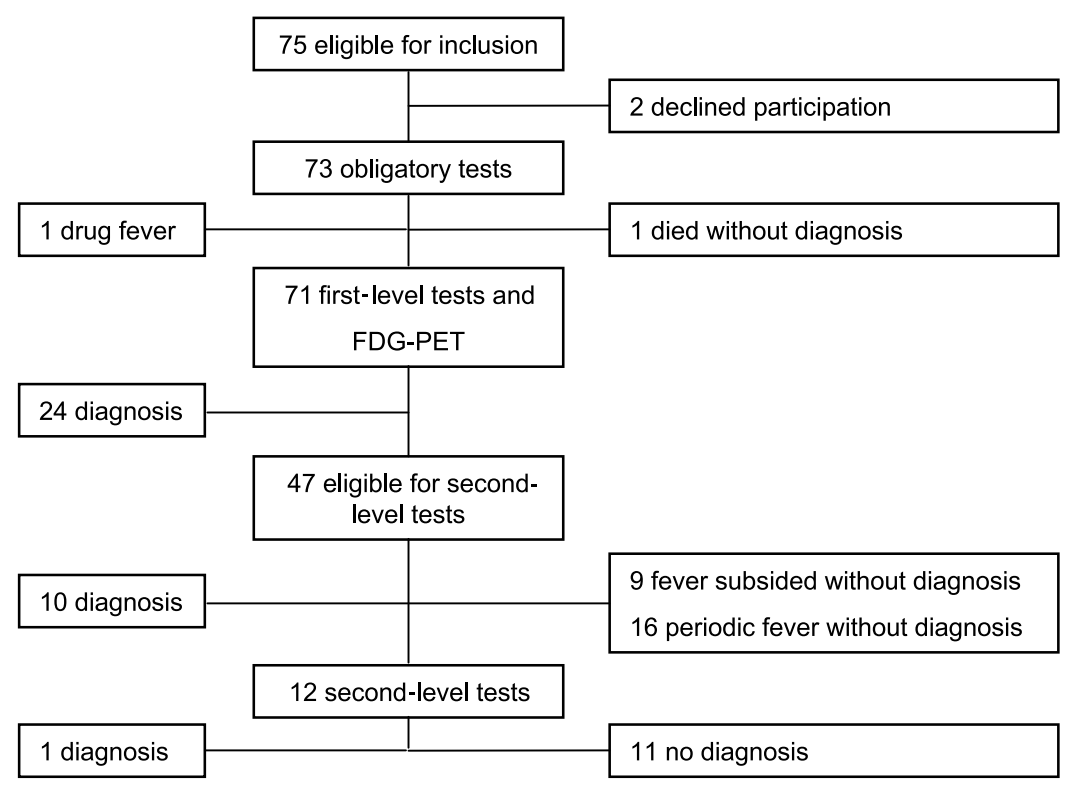

FIGURE 2. Number of patients included in the different stages of the diagnostic protocol, showing number of patients in whom a diagnosis was reached after every level of diagnostic tests, and, for the remaining patients, reasons for not participating in the next step of the diagnostic protocol. 
TABLE 1. Epidemiologic Data of All Patients With FUO

\begin{tabular}{|c|c|c|c|}
\hline Characteristic & $\begin{array}{c}\text { Total Patients } \\
(n=73) \text { No. }(\%)\end{array}$ & $\begin{array}{c}\text { University Hospital } \\
(n=40) \text { No. }(\%)\end{array}$ & $\begin{array}{l}\text { Community Hospitals } \\
(\mathrm{n}=33) \text { No. }(\%)\end{array}$ \\
\hline \multicolumn{4}{|l|}{ Patients per hospital } \\
\hline Radboud University Nijmegen Medical Centre & $40(55)$ & 40 & - \\
\hline Canisius-Wilhelmina Hospital & $11(15)$ & - & $11(33)$ \\
\hline Jeroen Bosch Hospital & $5(7)$ & - & $5(15)$ \\
\hline Rijnstate Hospital & $4(5)$ & - & $4(12)$ \\
\hline Slingeland Hospital & $11(15)$ & - & $11(33)$ \\
\hline Maxima Medical Centre & $2(3)$ & - & $2(6)$ \\
\hline \multicolumn{4}{|l|}{ Age, in years } \\
\hline Mean $\pm \mathrm{SD}$ & $54 \pm 16$ & $51 \pm 16$ & $57 \pm 16$ \\
\hline Range & $26-87$ & $26-82$ & $31-87$ \\
\hline Male sex & $33(45)$ & $16(40)$ & $17(52)$ \\
\hline Secondary referral & $15(21)$ & $15(38)^{*}$ & $0^{*}$ \\
\hline Periodic fever & $25(34)$ & $20(50)^{*}$ & $5(15)^{*}$ \\
\hline \multicolumn{4}{|l|}{ Duration of fever before present analysis, in days } \\
\hline Median \pm SD & $53 \pm 890$ & $348 \pm 1106^{*}$ & $15 \pm 200^{*}$ \\
\hline Range & $1-4197$ & $1-4197$ & $1-1144$ \\
\hline Hospitalization, no. of patients (\%) & $62(85)$ & $33(83)$ & $29(88)$ \\
\hline Median duration, in days $\pm \mathrm{SD}$ & $29 \pm 32$ & $32 \pm 40$ & $28 \pm 18$ \\
\hline Range, in days & $3-177$ & $3-177$ & $3-77$ \\
\hline
\end{tabular}

microbiology, or generally accepted clinical criteria ${ }^{14,16,18,29}$ in 31 cases $(86 \%)$. A probable confirmation was reached in the remaining 5 cases $(14 \%)$ through radiology and clinical follow-up. In 37 patients (51\%), the cause of the fever was not found. The percentage of patients without a final diagnosis did not differ significantly between RUNMC and the community hospitals (55\% vs. $45 \%$ ). In 19 of all 25 patients with periodic fever $(76 \%)$, no cause of the fever was found. Of the remaining 48 patients with continuous fever, no diagnosis was established in 15 cases $(31 \%, \mathrm{p}<0.005)$. In patients referred for a second opinion, no diagnosis was reached in 17 of 25 patients $(68 \%)$ compared to no diagnosis in 20 of 48 patients $(42 \%)$ who were directly referred $(\mathrm{p}=0.05)$.

After a median follow-up of 12 months (range, 6-23 mo), the fever subsided spontaneously in 16 of all 37 patients without a final diagnosis; the fever subsided after empirical treatment with a nonsteroidal antiinflammatory drug (NSAID) or corticosteroids in 5 patients; and in 15 patients, the fever persisted. Of these 15 patients with persisting fever after a median follow-up of 15 months (range, 7-23 mo), 10 had periodic fever. One patient died before a diagnosis was reached. Duration of follow-up exceeded 6 months in all surviving patients without a diagnosis. In 36 patients with an explanation for the fever and a median follow-up of 11 months (range, 4-23 mo for surviving patients, $>6$ mo in $88 \%$ ), the fever subsided either spontaneously or after specific treatment in 22 patients, and in 10 patients the fever persisted. Despite appropriate treatment, 4 patients died due to the underlying disease causing the fever (pulmonary infection with bronchiectasis, abdominal abscesses, non-Hodgkin lymphoma [NHL], and metastatic adenocarcinoma).

\section{Potentially Diagnostic Clues}

After the standardized history, physical examination, and obligatory tests, PDCs were present in all patients (Table 3). On average, 15 PDCs were identified per patient, of which $81 \%$ proved to be misleading. The remaining $19 \%$ of PDCs contributed to the final diagnosis, but PDCs alone did not directly lead to a diagnosis in any case. In all patients with PDCs contributing to the final diagnosis, misleading PDCs were also present. The PDCs most often leading to a diagnosis ( $<75 \%$ misleading, present in at least 10 patients) were weight loss, muscle weakness, skin changes, previous medical history, shortness of breath, chest pain, abdominal pain, arthralgia, morning stiffness, abnormal pulmonary auscultation, elevated LDH, anemia, leukocytosis, abnormal urinalysis, and abnormal chest X-ray. There was no significant difference between patients with a diagnosis and patients without a diagnosis or between patients from RUNMC and patients from the community hospitals regarding the number of PDCs present.

\section{Adherence to the Diagnostic Protocol}

A total of 5331 investigations were performed in these 73 patients. Adherence to the diagnostic protocol did not differ significantly between the university hospital and the community hospitals except for measurement of cryoglobulins, which was significantly more often performed in the university hospital than in the community hospitals $(95 \%$ 
TABLE 2. Final Diagnoses in 73 Patients With FUO

\begin{tabular}{|c|c|c|c|}
\hline Category & $\begin{array}{c}\text { Total Patients } \\
(n=73) \text { No. }(\%)\end{array}$ & $\begin{array}{c}\text { University Hospital } \\
(n=40) \text { No. }(\%)\end{array}$ & $\begin{array}{c}\text { Community Hospitals } \\
(\mathrm{n}=33) \text { No. }(\%)\end{array}$ \\
\hline Infection & $12(16)$ & $7(18)$ & $5(15)$ \\
\hline Bronchiectasia/pneumonia & 2 & 1 & 1 \\
\hline Diverticulitis & 1 & 1 & - \\
\hline Pyelonephritis & 1 & - & 1 \\
\hline Abdominal abscess & 1 & 1 & - \\
\hline Osteomyelitis & 2 & 1 & 1 \\
\hline Tonsillitis & 1 & - & 1 \\
\hline Chronic persistent yersiniosis & 4 & 3 & 1 \\
\hline Neoplasm & $5(7)$ & $2(5)$ & $3(9)$ \\
\hline Non-Hodgkin lymphoma & 3 & 2 & 1 \\
\hline Breast carcinoma with metastases & 1 & - & 1 \\
\hline Adenocarcinoma with unknown primary & 1 & - & 1 \\
\hline Noninfectious inflammatory disease & $16(22)$ & $8(20)$ & $8(24)$ \\
\hline Large vessel vasculitis & 2 & - & 2 \\
\hline Polymyalgia rheumatica & 3 & 1 & 2 \\
\hline Henoch-Schönlein purpura & 1 & 1 & - \\
\hline Microscopic polyangiitis & 1 & 1 & - \\
\hline Psoriatic arthritis & 1 & - & 1 \\
\hline Adult-onset Still disease & 3 & 2 & 1 \\
\hline Systemic lupus erythematosus & 4 & 2 & 2 \\
\hline Cryoglobulinemia & 1 & 1 & - \\
\hline Miscellaneous & $3(4)$ & 0 & $3(9)$ \\
\hline Drug fever & 2 & - & 2 \\
\hline Hypertriglyceridemia & 1 & - & 1 \\
\hline No diagnosis & $37(51)$ & $22(55)$ & $15(45)$ \\
\hline Spontaneous recovery & 16 & 6 & 10 \\
\hline Recovery with NSAID or corticosteroids & 5 & 3 & 2 \\
\hline Persistent fever & 15 & 12 & 3 \\
\hline Death & 1 & 1 & - \\
\hline
\end{tabular}

vs. $52 \%$ ). The obligatory investigations were performed in more than $95 \%$ of cases except for creatine phosphokinase $(\mathrm{n}=66,90 \%)$ and tuberculin skin test $(\mathrm{n}=58,79 \%)$. After the obligatory tests, 1 patient was diagnosed with drug fever (see Figure 2). After performing FDG-PET and testing for cryoglobulins, a diagnosis was reached in 24 patients. In 10 patients, new PDCs leading to the final diagnosis (systemic lupus erythematosus [SLE] in 2 patients; polymyalgia rheumatica in 3 patients; adult-onset Still disease in 2 patients; and microscopic polyangiitis, Henoch-Schönlein purpura, and hypertriglyceridemia in 1 patient each) developed before second-level tests were completed. In 9 patients, the fever subsided spontaneously and 16 patients had periodic fever, so only 12 patients with continuous fever qualified for secondlevel tests. Pyelonephritis was diagnosed with abdominal CT in 1 of these 12 patients, after which no further tests were performed. Abdominal CT was performed in 9 of the remaining 11 patients $(82 \%)$, thoracic CT in 7 patients $(64 \%)$, funduscopy in 4 patients $(36 \%)$, and bone marrow biopsy in only 1 patient (9\%). In 2 of the 4 patients older than 55 years, temporal artery biopsy was performed. An explanation for the fever was not found in any of the 11 patients.

\section{Utility of Investigations in the Diagnostic Process}

\section{Laboratory Investigations}

Including the obligatory laboratory tests, other chemical tests, immunologic serology, and endocrinologic investigations, a total of 3124 laboratory tests were performed (repeated measurements of the same parameter in 1 patient were not counted). None of these tests directly revealed the diagnosis. Abnormal liver tests, present in 20 patients $(27 \%)$, contributed to the final diagnosis in only 3 patients $(4 \%)$. The presence of antinuclear antibodies was helpful in diagnosing SLE in all 4 patients diagnosed with this disease, but showed false positive results in 8 patients. In 1 patient with microscopic polyangiitis, the presence of antineutrophil cytoplasmic antibodies (ANCA) was helpful, while ANCA were also 
TABLE 3. Number of PDCs Derived From the Standardized History, Physical Examination, and Obligatory Tests in 73 Patients With FUO

\begin{tabular}{lccc}
\hline How PDC Identified & $\begin{array}{c}\text { Total Patients }(\mathbf{n}=\mathbf{7 3}) \\
\text { No. of PDCs (\%) }\end{array}$ & $\begin{array}{c}\text { University Hospital (n= 40) } \\
\text { No. of PDCs (\%) }\end{array}$ & $\begin{array}{c}\text { Community Hospitals (n }=33) \\
\text { No. of PDCs (\%) }\end{array}$ \\
\hline History & 649 & 354 & 295 \\
Misleading & $540(83)$ & $297(84)$ & $243(82)$ \\
Physical examination & 128 & 64 & 64 \\
Misleading & $92(72)$ & $45(70)$ & $47(73)$ \\
Obligatory tests & 287 & 140 & 147 \\
Misleading & $225(78)$ & $108(77)$ & $117(80)$ \\
Total & 1064 & 558 & 506 \\
Misleading & $857(81)$ & $450(81)$ & $407(80)$ \\
\hline
\end{tabular}

present in 5 patients without vasculitis. Endocrinologic investigations did not contribute to the final diagnosis in any of the patients.

\section{Microbiology}

In 53 patients, 509 microbiologic serologic tests were performed (repeated measurements of the same parameter were not counted). Except for Yersinia enterocolitica serology, these tests were never helpful in establishing a diagnosis. Serology tests for cytomegalovirus $(n=37)$, Epstein-Barr virus $(\mathrm{n}=34)$, hepatitis B virus $(\mathrm{n}=21)$, hepatitis C virus $(\mathrm{n}=23)$, HIV $(\mathrm{n}=22)$, Borrelia burgdorferi $(\mathrm{n}=22)$, Brucella species $(\mathrm{n}=25)$, Coxiella burnetii $(\mathrm{n}=22)$, Toxoplasma gondii $(\mathrm{n}=25)$, and syphilis $(\mathrm{n}=21)$ were most often requested. In 29 patients (40\%), serology tests for Yersinia enterocolitica were performed. Yersinia serology results were negative in 15 patients, and were considered equivocal in 10 patients (IgA negative and $\mathrm{IgG}$ positive for at least 2 bands, IgA and IgG positive for 1 band, or IgA and IgG weakly positive for 1 or more bands). Positive results (IgA and $\mathrm{IgG}$ repeatedly positive for at least 2 bands) were obtained in 4 patients who were diagnosed with chronic persistent yersiniosis as cause of the fever. In 3 patients, all symptoms disappeared after antibiotic treatment and in 1 patient, symptoms disappeared and inguinal lymphadenopathy markedly decreased without treatment.

A total of 743 blood cultures, 170 urine cultures, and 280 other cultures were performed. In none of these patients, however, did blood or urine cultures lead to the cause of the fever. In 10 patients positive blood cultures were found (1.2\% of all blood cultures). In 1 patient treated with continuous ambulatory peritoneal dialysis (CAPD) who was diagnosed with abdominal abscesses, Escherichia coli, Proteus mirabilis, and Enterococcus species were repeatedly cultured from the CAPD fluid, which proved to be the microorganisms causing the abscesses after surgical drainage. In 6 patients, blood cultures grew coagulase-negative Staphylococcus species $(\mathrm{n}=3)$, Corynebacterium $(\mathrm{n}=1)$, diphtheroid rods $(n=1)$, and Propionibacterium $(n=1)$, which were all considered to be contamination by the treating physician. In the remaining 3 patients with blood cultures growing Streptococcus viridans, Stenotrophomonas maltophilia, and Enterococcus species combined with coagulase-negative Staphylococcus species, respectively, the fever persisted after appropriate antibiotic treatment followed by negative blood cultures and was considered to be unrelated to the bacteremia. In 9 patients who did not have convincing symptoms or signs of a urinary tract infection, urinary culture results were considered to be false positive (5\% of all urine cultures), because after adequate treatment of the assumed urinary tract infection, bacteriuria disappeared while the fever remained unchanged. In 1 patient eventually diagnosed with pyelonephritis, urinary culture (obtained after 2 days of antibiotic treatment) was considered false negative. With regard to the number of cultures performed per patient, no difference was found between the university hospital and the community hospitals.

\section{Imaging Techniques (Table 4)}

Chest X-ray was considered false negative in 4 patients with pulmonary NHL, mediastinal NHL, and SLE pleuritis $(\mathrm{n}=2)$. Abdominal ultrasound was performed in 58 patients (79\%). An abdominal CT had been performed in 6 of the remaining patients before inclusion or referral to the university hospital. In the other 9 patients, abdominal CT was preferred by the treating physician, usually because of a longer waiting time for abdominal ultrasound. In 18 patients, abnormal abdominal ultrasound did not lead to the diagnosis and caused unnecessary investigations, so these were considered false positive. In 1 patient with diverticulitis, abdominal ultrasound was classified as false negative because FDG-PET and subsequent abdominal CT revealed the diagnosis. Assuming all other normal chest X-rays and abdominal ultrasounds were true negative, we calculated sensitivity and specificity (Table 4).

Sinus X-rays and orthopantomograms were not helpful in our patient population. The results of 2 sinus X-rays and 2 orthopantomograms were considered false positive. Also, barium enemas or enteroclysis never helped reveal a final diagnosis in this study. In 1 patient, barium enema showed diverticulosis and could not rule out diverticulitis, but abdominal CT and colonoscopy did not reveal any signs of diverticulitis, so barium enema was considered false positive. In this population of patients with FUO, echocardiography 
TABLE 4. Diagnostic Utility of Imaging Techniques, Endoscopy, Histologic Investigations, and Laparotomy in 73 Patients With FUO

\begin{tabular}{|c|c|c|c|c|c|}
\hline Investigation & No. of Patients & Helpful & False Positive & \% Sensitivity (95\% CI) & $\%$ Specificity $(95 \%$ CI $)$ \\
\hline Chest X-ray & $73(100)$ & $6(8)$ & $8(11)$ & $60(26-88)$ & $87(77-94)$ \\
\hline Sinus X-ray & $32(44)$ & 0 & $2(6)$ & $\mathrm{NC}$ & $\mathrm{NC}$ \\
\hline Orthopantomogram & $22(30)$ & 0 & $2(9)$ & $\mathrm{NC}$ & $\mathrm{NC}$ \\
\hline Barium enema & $10(13,7)$ & 0 & $1(10)$ & $\mathrm{NC}$ & $\mathrm{NC}$ \\
\hline Enteroclysis & $6(8)$ & 0 & 0 & $\mathrm{NC}$ & $\mathrm{NC}$ \\
\hline Abdominal ultrasound & $58(79)$ & $6(10)$ & $18(31)$ & $86(42-100)$ & $65(50-78)$ \\
\hline Abdominal CT & $60(82)$ & $12(20)$ & $17(28)$ & $92(64-100)$ & $63(48-77)$ \\
\hline Chest CT & $46(63)$ & $9(20)$ & $8(17)$ & $82(48-98)$ & $77(60-90)$ \\
\hline FDG-PET & $70(96)$ & $23(33)$ & $10(14)$ & $92(74-99)$ & $78(63-89)$ \\
\hline Echocardiography & $19(26)$ & 0 & $4(22)$ & $\mathrm{NC}$ & $\mathrm{NC}$ \\
\hline Bronchoscopy & $5(7)$ & $1(20)$ & 0 & $\mathrm{NC}$ & $\mathrm{NC}$ \\
\hline Gastroscopy & $21(29)$ & 0 & $3(14)$ & $\mathrm{NC}$ & $\mathrm{NC}$ \\
\hline Colonoscopy & $19(26)$ & $1(5)$ & $2(10)$ & $\mathrm{NC}$ & $\mathrm{NC}$ \\
\hline Temporal artery biopsy & $14(19)$ & $1(7)$ & 0 & $\mathrm{NC}$ & $\mathrm{NC}$ \\
\hline Bone marrow biopsy & $19(26)$ & $2(11)$ & $1(5)$ & $\mathrm{NC}$ & $\mathrm{NC}$ \\
\hline Liver biopsy & $7(10)$ & $1(14)$ & $3(43)$ & $\mathrm{NC}$ & $\mathrm{NC}$ \\
\hline Duodenal biopsy & $12(16)$ & 0 & $1(8)$ & $\mathrm{NC}$ & $\mathrm{NC}$ \\
\hline Colonic biopsy & $13(18)$ & 0 & $2(15)$ & $\mathrm{NC}$ & $\mathrm{NC}$ \\
\hline Lymph node biopsy & $11(15)$ & $5(46)$ & $3(27)$ & $\mathrm{NC}$ & $\mathrm{NC}$ \\
\hline Skin biopsy & $8(11)$ & $5(63)$ & 0 & $\mathrm{NC}$ & $\mathrm{NC}$ \\
\hline Laparotomy & $4(6)$ & $2(50)$ & $1(25)$ & $\mathrm{NC}$ & $\mathrm{NC}$ \\
\hline
\end{tabular}

Abbreviations: $\mathrm{CI}=$ confidence intervals; $\mathrm{NC}=$ not calculated.

was never helpful. In 4 patients without cardiac causes explaining the fever, abnormal echocardiography showing a small pericardial effusion in the patient with diverticulitis, dubious vegetations of the mitral or aortic valve without endocarditis according to the Duke criteria $(n=2)$, and thickened septum was considered false positive.

Abdominal CT, performed in 60 patients, was helpful in establishing a diagnosis in 12 patients (20\%). Results of abdominal CT were considered false positive in 17 patients (28\%, 12 patients without a final diagnosis and 5 patients with a diagnosis not explaining the abnormality). Abdominal CT was considered false negative in 1 patient who was eventually diagnosed with diverticulitis; in that patient only the last of 3 abdominal CT scans was considered abnormal after comparison to the FDG-PET results. In 46 patients, chest CT was performed, which was helpful in $9(20 \%)$. False positive results were found in 8 patients without evidence of pulmonary disease (17\%). Chest CT was considered false negative in a patient with NHL and in a patient with aortitis of the thoracic aorta. FDG-PET, performed in 70 patients, contributed to the final diagnosis in 23 patients $(33 \%)$, and results were categorized as false positive in 10 patients (14\%, 9 patients without a diagnosis). FDG-PET was considered false negative in 2 patients $(3 \%)$ with small pleural effusions who were eventually diagnosed with SLE. The results of FDG-PET will be discussed in detail elsewhere. Assuming all other abdominal and chest CT-scans and FDG-PET-scans were true negative, we calculated sensitivity and specificity (see Table 4).

\section{Histologic Investigations}

Bone marrow aspiration was performed in 21 patients, but never contributed to the diagnosis. Bone marrow biopsy, performed in 19 patients, was helpful in establishing a diagnosis in 1 patient with NHL and 1 patient with bone metastases of an adenocarcinoma with an unknown primary tumor. In both patients, bone marrow aspiration had not revealed the diagnosis. Temporal artery biopsy was performed in 14 patients (10 with PDCs for vasculitis, 2 with FDG-PET results suggesting vasculitis, and 2 as part of the second-level tests), but was useful to confirm the diagnosis of temporal arteritis in only 1 patient, in whom FDG-PET already pointed to large vessel vasculitis. Liver biopsy was performed in 7 patients. In 1 patient, lobular hepatitis supported the suspected diagnosis of persistent yersiniosis and thus contributed somewhat to the diagnosis. In 3 patients, the results were considered false positive: hepatitis suggestive of a drug reaction in the patient with diverticulitis and nonspecific hepatitis and steatosis in 2 patients without a diagnosis and no signs of progressive liver disease or viral hepatitis after follow-up of 8 and 11 months, respectively. Lymph node biopsy was performed in 11 patients with lymphadenopathy. Lymph node biopsy supported persistent yersiniosis in 3 patients. Reactive changes were found in 3 patients without a final diagnosis (considered to be false positive) and in 2 patients with Still disease (true positive). Skin biopsy, performed in 8 patients with skin changes, was helpful in 5 patients diagnosed with drug fever $(\mathrm{n}=2)$, Henoch-Schönlein purpura, SLE, and Still 
disease. Other successful histologic investigations performed because PDCs were present or because of abnormal FDGPET results were pulmonary wedge excision, confirming infection of known bronchiectasis, SLE, and NHL in 1 patient each; histologic examination of an excised tonsil confirming tonsillitis in 1 patient; and biopsy of the peritoneum showing a granulomatous infiltrate with necrosis supporting the diagnosis of persistent yersiniosis in 1 patient. Autopsy confirmed the presence of NHL in 1 patient.

\section{Endoscopy and Laparotomy or Laparoscopy}

Bronchoscopy was performed in only 5 patients and supported the diagnosis in 1 patient eventually diagnosed with acute infection of bronchiectasis after pulmonary wedge excision. Gastroscopy, performed in 21 patients, and colonoscopy, performed in 19 patients, were never useful. Gastroscopy showed false positive results in 3 patients (ischemic changes, duodenal ulceration and bulbitis, polyp suspected for malignancy). Colonoscopy was false positive in 2 patients (rectal ulceration and local redness with normal biopsy results). Laparotomy or laparoscopy was performed in 4 patients with PDCs of abdominal pathology: in 1 patient, lymphadenopathy caused by persistent yersiniosis was confirmed and in 1 patient peritonitis was found, which was later diagnosed as SLE peritonitis. In 1 patient, laparotomy showed lymphadenopathy, but biopsy showed only reactive changes and follow-up of almost 1 year has not revealed a diagnosis, so laparotomy was considered false positive.

\section{Factors Predicting the Likelihood of Reaching a Diagnosis}

Statistically significant predictors for reaching a diagnosis are shown in Table 5. The chance of establishing a diagnosis was higher in patients with continuous fever and in patients in whom the fever persisted for less than 180 days before inclusion. Foreign descent appeared to be a significant predictor of reaching a diagnosis, although these patients were diagnosed with diseases (SLE and large vessel vasculitis) that do not appear to relate to their ethnicity. Surprisingly, otalgia was also a significant predictor. Two of these patients were diagnosed with large vessel vasculitis, which could explain the otalgia, but the 5 remaining patients were diagnosed with diseases not explaining the otalgia (adult-onset Still disease, hypertriglyceridemia, pneumonia, NHL, and psoriatic arthritis, respectively). In none of 11 patients with normal CRP and normal ESR, could a diagnosis be reached compared to 36 of 62 patients with elevated CRP and/or ESR $(58 \%, \mathrm{p}<0.0005)$, so elevated ESR or CRP is also an important factor in predicting the likelihood of reaching a diagnosis. Abnormal results of either chest X-ray, abdominal ultrasound, or abdominal CT were not predictive of reaching a diagnosis. Abnormal chest CT (performed in 46 patients) and abnormal FDG-PET (performed in 70 patients), however, were significant predicting factors.

\section{DISCUSSION}

In this prospective multicenter study of 73 patients recruited from 1 university hospital and 5 community hospitals, the proportion of undiagnosed cases (51\%) seems high, but the percentage of undiagnosed cases tends to increase in more recent studies. In the only 2 series of patients with classical FUO studied after 1990 in northwestern Europe, no diagnosis was reached in $30 \%{ }^{10}$ and $53 \%{ }^{35}$, respectively. There are several possible explanations. First, $35 \%$ of all patients were referred after extensive investigations elsewhere, and it has been speculated that more difficult-to-diagnose cases are referred. The percentage of referred patients in this study, however, is similar to other recent studies (Table 6) reporting percentages of undiagnosed cases from $11 \%$ to $53 \%$, $7,10,12,25,35$. Furthermore, in the present study, secondary referral was not a significant factor predicting the likelihood of reaching a diagnosis. A more probable explanation is the diagnostic strictness we, and also others with relatively high percentages of undiagnosed $\operatorname{cases}^{7,10,25,35}$, have applied. Diagnoses lacking persuasive confirmatory tests (for example, adult-onset Still disease, polymyalgia rheumatica) were accepted only if

TABLE 5. Predictors of the Likelihood of Reaching a Diagnosis in 73 Patients With FUO

\begin{tabular}{lccr}
\hline Parameter & $\begin{array}{c}\text { No Diagnosis }(\mathbf{n}=\mathbf{3 7}) \\
\text { No./Total No. }(\mathbf{\%})\end{array}$ & $\begin{array}{c}\text { Diagnosis }(\mathbf{n}=\mathbf{3 6}) \\
\text { No./Total No. (\%) }\end{array}$ & \begin{tabular}{c} 
RR (95\% CI) \\
\hline Continuous fever
\end{tabular} \\
Fever present $<180$ days* $^{\dagger}$ & $18 / 37(49)$ & $30 / 36(83)$ & $2.6(1.3-5.4)$ \\
Foreign descent $^{\dagger}$ & $18 / 37(49)$ & $31 / 36(86)$ & $3.0(1.4-6.8)$ \\
Otalgia & $0 / 37$ & $2 / 36(6)$ & $2.6(1.1-6.4)$ \\
Elevated CRP or ESR & $1 / 37(3)$ & $7 / 36(19)$ & $2.0(1.3-2.9)$ \\
Elevated LDH & $26 / 37(70)$ & $36 / 36(100)$ & - \\
Leukopenia & $2 / 37(5)$ & $9 / 36(25)$ & $1.9(1.3-2.8)$ \\
Thrombocytosis & $0 / 37$ & $2 / 36(6)$ & $2.1(1.6-2.7)$ \\
Chest CT abnormal & $2 / 37$ & $7 / 36(19)$ & $1.7(1.1-2.7)$ \\
FDG-PET abnormal & $6 / 26(23)$ & $12 / 20(60)$ & $2.3(1.2-4.6)$ \\
\hline
\end{tabular}

*Fever present $<180$ days before inclusion in the study.

${ }^{\dagger}$ Either the patient or 1 or both parents were not born in The Netherlands.

${ }^{\ddagger}$ Calculation of relative risk not possible because all patients with a diagnosis had an elevated CRP or ESR. 
TABLE 6. Comparison of Previous FUO Series With Inclusion of Patients Since 1980 from Europe and the United States

\begin{tabular}{|c|c|c|c|c|c|c|c|c|c|}
\hline $\begin{array}{c}\text { First Author } \\
\text { [Ref.] (Country) }\end{array}$ & Design & $\begin{array}{c}\text { No. of Patients } \\
\text { (Recruitment } \\
\text { Period) }\end{array}$ & $\begin{array}{l}\text { Secondary } \\
\text { Referral } \\
(\%)\end{array}$ & $\begin{array}{l}\text { Periodic } \\
\text { Fever } \\
(\%)\end{array}$ & \multicolumn{5}{|c|}{ Cause of Fever (\%) } \\
\hline Knockaert $^{25}$ (Belgium) & Prospective $1 \mathrm{UH}$ & 199 (1980-1989) & 28 & & 23 & 7 & 25 & 20 & 26 \\
\hline Barbado $^{2}$ (Spain) & Prospective $1 \mathrm{UH}$ & 85 (1982-1989) & 45 & & 11 & 28 & 34 & 12 & 15 \\
\hline Kazanjian $^{19}$ (US) & Prospective $3 \mathrm{CH}$ & 86 (1984-1990) & & & 33 & 24 & 26 & 5 & 9 \\
\hline De Kleijn ${ }^{7}$ (NL) & Retrospective $1 \mathrm{UH}$ & $53(1988-1992)$ & 53 & 34 & 21 & 19 & 23 & 8 & 30 \\
\hline Ergonul $^{12}$ (Turkey) & Prospective $1 \mathrm{UH}$ & 80 (1993-1999) & 33 & & 52 & 18 & 16 & 3 & 11 \\
\hline $\begin{array}{l}\text { Vanderschueren } \\
\text { (Belgium) }\end{array}$ & Prospective $1 \mathrm{UH}$ & 185 (1990-1999) & 32 & 42 & 11 & 10 & 18 & 8 & 53 \\
\hline Saltoglu ${ }^{33}$ (Turkey) & Prospective $1 \mathrm{UH}$ & 87 (1994-2002) & & & 59 & 14 & 18 & 2 & 7 \\
\hline Present report (NL) & Prospective $1 \mathrm{UH} / 5 \mathrm{CH}$ & $73(2003-2005)$ & 34 & 34 & 16 & 7 & 22 & 4 & 51 \\
\hline
\end{tabular}

Abbreviations: $\mathrm{UH}=$ university hospital, $\mathrm{CH}=$ community hospital, US $=$ United States, NL $=$ The Netherlands, misc $=$ miscellaneous.

sufficient standard criteria were met and follow-up allowed exclusion of other diseases. Since periodic fever was strongly related to a reduced chance of reaching a diagnosis in the current study and the chance of reaching a diagnosis in these patients is known to be significantly smaller than in patients with continuous fever ${ }^{10,24,35}$, the high percentage of patients with periodic fever (34\%) appears to be an important factor explaining the high number of undiagnosed cases. Another factor contributing to the seemingly high diagnostic failure rate might be that a diagnosis is more frequently reached before 3 weeks have elapsed because patients with fever tend to seek medical advice earlier and because better diagnostic techniques, such as CT or magnetic resonance imaging, are widely available resulting in more hard-to-diagnose cases meeting the definition of FUO. Another contributing factor, as suggested by Vanderschueren et $\mathrm{al}^{35}$, could be the observation that most patients with FUO without a diagnosis do well ${ }^{10,21}$, which may lead to a less aggressive diagnostic approach in clinically stable patients once diseases with immediate therapeutic or prognostic consequences have been ruled out to a reasonable extent. This could be especially true for patients with periodic fever who are asymptomatic in between febrile episodes. In the current study, the outcome of patients without a diagnosis was as favorable as in previous studies ${ }^{10,21,35}$.

The spectrum of diseases now considered to cause FUO has changed as a result of changes in the broad spectrum of diseases causing FUO and the availability of new diagnostic techniques. The proportion of abdominal abscesses and tumors, for example, has decreased in recent series because of earlier detection by ultrasound. Infective endocarditis has decreased in frequency as blood culture techniques have improved. Some diagnoses in recent series, such as Lyme disease, acute HIV infection, and Sweet syndrome, were unknown 4 decades ago. The percentage of patients diagnosed with infection in the current study is similar to the results of other recent studies performed in northwestern Europe ${ }^{7,10,25,35}$ (see Table 6). In most studies from southern Europe ${ }^{12,33,34}$, infections account for a much larger proportion of diagnoses $(34 \%-59 \%)$, which can be explained by the high incidence of tuberculosis $(29 \%-70 \%$ of all infections). In the only study from southern Europe with a lower percentage of infections ${ }^{2}, 8$ of 9 infections were caused by tuberculosis also. In 1 study from the United States $^{19}$, the percentage of infections was also higher, which could possibly be explained by not excluding HIV-patients and by selection of FUO cases from all infectious disease consultations instead of from the general patient population. The patients in the current study did not suffer from rare infections but exhibited rather atypical manifestations of common illnesses, which has already been emphasized as an important factor in patients with FUO by Petersdorf and Beeson $^{31}$. The number of patients eventually diagnosed with NIID will probably not decrease in the near future, because fever may precede more typical manifestations or serologic evidence by months in these diseases. Moreover, many of NIID can only be diagnosed after prolonged observation and exclusion of other diseases. In other recent series, the proportion of patients diagnosed with malignancy roughly varies between $10 \%$ and $20 \%$. In 2 studies, a higher incidence of malignancy was found, but both of these studies had a remarkably low percentage of patients without a diagnosis suggesting different patient characteristics ${ }^{2,19}$. The widespread early use of diagnostic techniques such as ultrasound and $\mathrm{CT}$ have resulted in a steady decline of malignancy explaining FUO.

To enable adequate comparison between FUO studies, using a uniform definition and uniform entry criteria is very important. Selection bias increases when patients presenting to the outpatient department are included, because prospective case finding is harder and standardized diagnostic protocols are more difficult to implement. However, inclusion of these patients in future studies, as we did in the current study, is essential. Since Petersdorf and Beeson first defined FUO, health care has shifted from the inpatient to the ambulatory setting. Today most patients with FUO will be 
hospitalized if this is required by their clinical condition, but not for diagnostic purposes only. A vast proportion of patients who met the definition of FUO in the past would be omitted from future studies if the requirement of study in the hospital is maintained (11 patients in our study, for example). Also, the criterion of no diagnosis after admission to the hospital for 1 week (or 3 days as was previously suggested by others) is a time-related criterion, which may cause important differences, as it is dependent on the experience of the physician. Furthermore, many differences in management and diagnostic facilities exist among hospitals or countries. We strongly recommend that, in future FUO studies, this criterion is changed to a qualityrelated criterion requiring a minimum list of certain investigations to be performed. Defining the necessary initial investigations will remain a matter of debate, but it is generally agreed that the initial diagnostic protocol required for a case to qualify as FUO should at least include the tests that were obligatory in our study. Further tests should be based on the local prevalence of certain diseases.

Classic test characteristics are difficult to apply in FUO studies since there is no diagnostic gold standard against which diagnostic tests may be measured. Determining the denominator for calculation of sensitivity and specificity is difficult since a significant proportion of all cases remain undiagnosed. Therefore, it seems more appropriate to rate diagnostic tests as useful-positive or helpful. The use of diagnostic tests in our patient population proved to be abundant. Although obligatory tests, the first-stage investigations, and FDG-PET were performed in more than 95\% of patients, the second-stage screening tests (see Figure 1) were completed in only 1 of 12 patients qualifying for this part of the protocol. Therefore, the current study does allow us to draw conclusions on the overall diagnostic value of many diagnostic techniques, but not, or to a lesser extent, on the screening diagnostic value of some of these techniques.

Only rarely do biochemical tests directly lead to a certain diagnosis. In the current study, nonspecific liver test abnormalities, present in $27 \%$ of all patients, only rarely contributed to the final diagnosis, which is in agreement with data from previous studies showing that abnormal liver tests are not predictive of a diagnostic liver biopsy in $\mathrm{FUO}^{17,28}$. The diagnostic yield of immunologic serology is also relatively low. Although antinuclear antibodies and ANCA sometimes contributed to the diagnosis, these tests were more often false positive and are of little use without PDCs pointing to specific immunologic disorders. Based on results from a larger prospective study ${ }^{9}$, the absence of specific symptoms in many patients, and the relatively low cost of the test, investigation of cryoglobulins appears to be a valuable screening test in patients with FUO, although in our current population, cryoglobulinemia was diagnosed in only 1 patient who was not suspected of this disease. De Kleijn et $\mathrm{al}^{9}$ studied the screening diagnostic value of microbiologic serology in 167 patients with FUO and concluded that these investigations should not be performed early in the diagnostic workup in patients without PDCs for specific infections. In the current study, serologic tests, although abundantly performed, contributed to the final diagnosis in only 4 patients with chronic yersiniosis, which supports that conclusion.

Although on average 16 cultures were performed in each patient, culture results supported the final diagnosis in only 1 patient. Of course, the obligation of the diagnosis being uncertain after performing at least 3 blood cultures and a urine culture before meeting the definition of FUO is an important reason for this. What physicians should learn from this, however, is that performing more than 3 blood cultures or more than 1 urine culture is useless in patients with FUO in the absence of PDCs (that is, a high clinical suspicion of endocarditis). In the current study, only 1 patient used antibiotics at the time of inclusion, when blood and urine cultures were performed, which might explain why additional blood cultures never contributed to the diagnosis in contrast to the study by De Kleijn et $\mathrm{al}^{9}$.

The diagnostic yield of imaging techniques is often difficult to establish, because it is dependent on the imaging techniques that are used in an earlier stage. We tried to minimize this problem by including chest X-ray and abdominal ultrasound in the obligatory part of the diagnostic protocol and by dividing the protocol in several stages. Abdominal ultrasound was chosen as an obligatory test above abdominal CT because of the relatively low cost, no radiation burden, and the absence of side effects. Of course, none of the patients in whom abdominal ultrasound or chest X-ray directly led to the diagnosis were included in the current study. In several patients meeting the criteria for FUO, however, abdominal ultrasound or chest X-ray contributed to the diagnosis. Despite the high number of false positive ultrasounds and the relatively low sensitivity of chest X-rays, we believe that these simple low-cost diagnostic tests remain obligatory in all patients with FUO in order to separate diseases that can be easily diagnosed from the real FUO cases.

In the current study, FDG-PET proved to be helpful in $33 \%$ of all patients. The value of FDG-PET in this patient population is discussed in greater detail in a separate article $\mathrm{e}^{3 \mathrm{a}}$ concluding that FDG-PET as part of a structured diagnostic protocol is valuable in the general population of FUO patients. The diagnostic yield of abdominal CT in our study $(20 \%)$ is similar to results from 2 previous studies investigating the usefulness of abdominal $\mathrm{CT}$ in $\mathrm{FUO}^{9,32}$. Specificity of chest CT was similar to the results from the study by De Kleijn et $\mathrm{al}^{9}$, but specificity of abdominal CT appeared to be lower (63\% vs. $80 \%)$. As stated above, this might be explained by the imaging techniques already used (FDG-PET in most patients in the current study vs. no FDGPET in the previous study). Despite the limited specificity of abdominal $\mathrm{CT}$ and the probably limited additional value of chest CT after normal FDG-PET, chest and abdominal CT may be used as screening procedures at a later stage of the diagnostic protocol due to their noninvasive nature and high sensitivity. In the current study as well as in the study by De Kleijn et $\mathrm{al}^{9}$, the diagnostic yield of echocardiography, $\mathrm{X}$-rays of the sinuses, radiologic or endoscopic evaluation of the gastrointestinal tract, and bronchoscopy were very low when PDCs were absent, so these tests should not be performed as screening procedures. 
Bone marrow aspiration was of no use in the absence of PDCs for bone marrow disorders in both the current study and the study by De Kleijn et $\mathrm{al}^{9}$, and is thus not recommended as a screening procedure. Bone marrow biopsy was useful in 2 patients without PDCs for bone marrow disorders in the current study, but in both patients FDG-PET already pointed to the diagnosis. With the addition of FDG-PET, which is very sensitive in detecting lymphoma, carcinoma, and osteomyelitis, the value of bone marrow biopsy as a screening procedure is probably less than in the study by De Kleijn et $\mathrm{al}^{9}$. Some studies have shown a high prevalence of temporal arteritis among patients with $\mathrm{FUO}^{25,35}$, up to $17 \%$ in elderly patients ${ }^{23}$. In the current study, temporal artery biopsy was useful in only 1 patient in whom vasculitis was already suspected after FDG-PET. Temporal artery biopsy can still be recommended for patients aged 55 years or older in a later stage of the diagnostic protocol, because FDG-PET will not be useful if the vasculitis is limited to the temporal arteries due to the small diameter of these vessels and because of high FDG-uptake in the overlying brain.

In the past, liver biopsies have often been performed as a screening procedure in patients with FUO. In 1 retrospective analysis of 24 patients with FUO, liver biopsy revealed 3 cases of histoplasmosis and 1 case of tuberculosis ${ }^{17}$, all very uncommon causes of FUO in Europe and the United States. In another study, liver biopsy was performed in 43 patients (14\% diagnostic), but these cases represented only $10 \%$ of the FUO patients seen in that hospital ${ }^{28}$, indicating considerable selection bias. Furthermore, in both the current study and the study by De Kleijn et $\mathrm{al}^{9}$, liver biopsy as part of the later stage of a screening diagnostic protocol was helpful in only 1 patient each. Liver biopsy is an invasive procedure with the possibility of complications and even death. Therefore, we believe that liver biopsy should not be performed as a screening procedure in all patients with FUO.

In many reviews of FUO since the 1970s, the importance of PDCs has been emphasized by advising the physician to observe Sutton's law "to go where the money is $" 5,13,22$. Two retrospective studies showed significantly lower chances of reaching a diagnosis when PDCs were absent $^{7,36}$. The value of PDCs was investigated in only 1 prospective study ${ }^{9}$, however, which showed that the presence of PDCs does not increase the likelihood of reaching a diagnosis. Because of the low percentage of patients without PDCs in that study and the absence of patients without PDCs in the current study, these findings should be interpreted carefully. We have no doubt that the search for PDCs is the physician's most important tool to unravel the cause of the fever, but both the current study and the study by De Kleijn et al show that most PDCs are misleading. In univariate analysis of patients with and patients without a diagnosis, significant predictive factors for reaching a diagnosis were continuous fever; duration of fever for less than 180 days; foreign descent; otalgia; elevated ESR, CRP, or LDH; leukopenia; thrombocytosis; abnormal chest CT; and abnormal FDG-PET. In the case of most of these factors, it is understandable why reaching a diagnosis is more likely, but especially in the case of "otalgia" and "foreign descent," it is much more difficult or even impossible, raising questions about the usefulness of these factors.

The vast majority of FUO studies have been performed in university hospitals. Patients with FUO are often referred to those institutions because of the local expertise, which is also true for RUNMC. It is questionable whether the results from such studies can be applied to the general population of patients with FUO. Two prospective studies were performed in community hospitals in the $1970 \mathrm{~s}^{15}$ and the $1980 \mathrm{~s}^{19}$, but, as far as we know, this is the first study directly comparing patients with FUO referred to 1 university hospital and several community hospitals in the same region during the same time. Gleckman et al ${ }^{15}$ studied 34 patients referred to 1 small community hospital (180 beds) in the Boston area. In $35 \%$ of these patients, no diagnosis was reached. In $18 \%$ of all cases, infection was causing the fever, in 9\% malignancy, in $12 \%$ NIID, and in $26 \%$ the cause was categorized as miscellaneous. In the more recent study by Kazanjian ${ }^{19}$, 86 patients with FUO were recruited from 3 community hospitals in Rhode Island (see Table 6). Diagnostic techniques have improved remarkably since Gleckman's study, and FDG-PET was added to the diagnostic possibilities since Kazanjian's study. In the current study, significantly more patients with periodic fever were found in the university hospital. Since diagnosis is more difficult in this patient population and our university hospital has specialized in periodic fever, this was to be expected. The fact that half of all patients were referred to RUNMC after extensive investigation elsewhere while in the community hospitals all patients were directly referred explains the significantly longer duration of fever before the present analysis. There were, however, no significant differences between the causes of FUO or the percentage of patients in whom no diagnosis was reached, so conclusions about the structured diagnostic protocol used in this study are applicable to the general population of FUO patients.

In conclusion, the proportions of patients with FUO that are eventually diagnosed with infection or malignancy have decreased in recent years, while the percentage of undiagnosed cases has increased significantly. In patients with FUO, empiric testing should be based on the relative frequencies of the different causes and their importance to the health of the patient. In the absence of PDCs for certain infectious diseases, serologic tests are nearly always useless. Except for tests from the obligatory part of our protocol, cryoglobulins in an early stage, followed by FDG-PET, and in a later stage by abdominal and chest $\mathrm{CT}$, temporal artery biopsy in patients aged 55 years or older, and possibly bone marrow biopsy, other tests should not be used as screening procedures in the hope that something abnormal will be found. Ordering other screening tests has many disadvantages, such as false positive results, the possibility of adverse reactions or complications, increasing costs of testing, and, as stated before by De Kleijn et $\mathrm{al}^{9}$, "a soporific effect on the doctor's diagnostic mental activities." Repeating a thorough history and physical examination and waiting for new PDCs to appear seems to be preferable to ordering more screening tests. If the fever persists and the source remains elusive after completing the 
later stage investigations, supportive treatment with NSAIDs can be helpful. Empirical therapeutic trials with antibiotics, steroids, or antituberculous agents should be avoided, except in patients whose condition is deteriorating.

\section{ACKNOWLEDGMENTS}

We thank Dr. G. Ferwerda (Rijnstate Hospital), Dr. A.W. van den Wall Bake (Maxima Medical Centre), and Dr. K. Simons (Maxima Medical Centre) for their help recruiting patients for this study.

\section{REFERENCES}

1. Arnow PM, Flaherty JP. Fever of unknown origin. Lancet. 1997; 350:575-580.

2. Barbado FJ, Vazquez JJ, Pena JM, et al. Pyrexia of unknown origin: changing spectrum of diseases in two consecutive series. Postgrad Med J. 1992;68:884-887.

3. Bleeker-Rovers CP, de Kleijn EM, Corstens FH, et al. Clinical value of FDG PET in patients with fever of unknown origin and patients suspected of focal infection or inflammation. Eur J Nucl Med Mol Imaging. 2004;31:29-37.

3a. Bleeker-Rovers $\mathrm{CP}$, Vos FJ, Mudde AH, et al. A prospective multicentre study of the value of FDG-PET as part of a structured diagnostic protocol in patients with fever of unknown origin. Eur J Nucl Med Mol Imaging. 2006 Dec. 14 [Epub ahead of print].

4. Blockmans D, Knockaert D, Maes A, et al. Clinical value of [(18)F]fluoro-deoxyglucose positron emission tomography for patients with fever of unknown origin. Clin Infect Dis. 2001;32:191-196.

5. Brusch JL, Weinstein L. Fever of unknown origin. Med Clin North Am. 1988;72:1247-1261.

6. Buysschaert I, Vanderschueren S, Blockmans D, et al. Contribution of (18)fluoro-deoxyglucose positron emission tomography to the work-up of patients with fever of unknown origin. Eur J Intern Med. 2004;15: $151-156$.

7. de Kleijn EM, van der Meer JW. Fever of unknown origin (FUO): report on 53 patients in a Dutch university hospital. Neth $J$ Med. 1995;47:54-60.

8. de Kleijn EM, van der Meer JW. Inquiry into the diagnostic workup of patients with fever of unknown origin. Neth J Med. 1997;50:69-74.

9. de Kleijn EM, van Lier HJ, van der Meer JW. Fever of unknown origin (FUO). II. Diagnostic procedures in a prospective multicenter study of 167 patients. The Netherlands FUO Study Group. Medicine (Baltimore). 1997;76:401-414.

10. de Kleijn EM, Vandenbroucke JP, van der Meer JW. Fever of unknown origin (FUO). I. A prospective multicenter study of 167 patients with FUO, using fixed epidemiologic entry criteria. The Netherlands FUO Study Group. Medicine (Baltimore). 1997;76:392-400.

11. Durack DT, Street AC. Fever of unknown origin - reexamined and redefined. Curr Clin Top Infect Dis. 1991;11:35-51.

12. Ergonul O, Willke A, Azap A, et al. Revised definition of "fever of unknown origin": limitations and opportunities. J Infect. 2005;50: $1-5$.

13. Esposito AL, Gleckman RA. A diagnostic approach to the adult with fever of unknown origin. Arch Intern Med. 1979;139:575-579.

14. Fautrel B, Zing E, Golmard JL, et al. Proposal for a new set of classification criteria for adult-onset Still disease. Medicine (Baltimore). 2002;81:194-200.

15. Gleckman R, Crowley M, Esposito A. Fever of unknown origin: a view from the community hospital. Am J Med Sci. 1977;274:21-25.

16. Hochberg MC. Updating the American College of Rheumatology revised criteria for the classification of systemic lupus erythematosus. Arthritis Rheum. 1997;40:1725.

17. Holtz T, Moseley RH, Scheiman JM. Liver biopsy in fever of unknown origin. A reappraisal. J Clin Gastroenterol. 1993;17:29-32.

18. Hunder GG, Bloch DA, Michel BA, et al. The American College of Rheumatology 1990 criteria for the classification of giant cell arteritis. Arthritis Rheum. 1990;33:1122-1128.

19. Kazanjian PH. Fever of unknown origin: review of 86 patients treated in community hospitals. Clin Infect Dis. 1992;15:968-973.

20. Kjaer A, Lebech AM, Eigtved A, et al. Fever of unknown origin: prospective comparison of diagnostic value of 18 F-FDG PET and 111In-granulocyte scintigraphy. Eur J Nucl Med Mol Imaging. 2004;31: 622-626.

21. Knockaert DC, Dujardin KS, Bobbaers HJ. Long-term follow-up of patients with undiagnosed fever of unknown origin. Arch Intern Med. 1996;156:618-620.

22. Knockaert DC, Vanderschueren S, Blockmans D. Fever of unknown origin in adults: 40 years on. $J$ Intern Med. 2003;253:263-275.

23. Knockaert DC, Vanneste LJ, Bobbaers HJ. Fever of unknown origin in elderly patients. J Am Geriatr Soc. 1993;41:1187-1192.

24. Knockaert DC, Vanneste LJ, Bobbaers HJ. Recurrent or episodic fever of unknown origin. Review of 45 cases and survey of the literature. Medicine (Baltimore). 1993;72:184-196.

25. Knockaert DC, Vanneste LJ, Vanneste SB, et al. Fever of unknown origin in the 1980s. An update of the diagnostic spectrum. Arch Intern Med. 1992;152:51-55.

26. Lorenzen J, Buchert R, Bohuslavizki KH. Value of FDG PET in patients with fever of unknown origin. Nucl Med Commun. 2001;22:779-783.

27. Meller J, Altenvoerde G, Munzel U, et al. Fever of unknown origin: prospective comparison of $[18 \mathrm{~F}] \mathrm{FDG}$ imaging with a double-head coincidence camera and gallium-67 citrate SPET. Eur J Nucl Med. 2000;27:1617-1625.

28. Mitchell DP, Hanes TE, Hoyumpa AM Jr, et al. Fever of unknown origin. Assessment of the value of percutaneous liver biopsy. Arch Intern Med. 1977;137:1001-1004.

29. Moll JM, Wright V. Psoriatic arthritis. Semin Arthritis Rheum. 1973; 3:55-78.

30. Petersdorf RG. Fever of unknown origin. An old friend revisited. Arch Intern Med. 1992;152:21-22.

31. Petersdorf RG, Beeson PB. Fever of unexplained origin: report on 100 cases. Medicine (Baltimore). 1961;40:1-30.

32. Quinn MJ, Sheedy PF, Stephens DH, et al. Computed tomography of the abdomen in evaluation of patients with fever of unknown origin. Radiology. 1980;136:407-411.

33. Saltoglu N, Tasova Y, Midikli D, et al. Fever of unknown origin in Turkey: evaluation of 87 cases during a nine-year-period of study. $J$ Infect. 2004:48:81-85.

34. Tabak F, Mert A, Celik AD, et al. Fever of unknown origin in Turkey. Infection. 2003;31:417-420.

35. Vanderschueren S, Knockaert D, Adriaenssens T, et al. From prolonged febrile illness to fever of unknown origin: the challenge continues. Arch Intern Med. 2003;163:1033-1041.

36. Wanvarie S, Tanphaichitra D, Limsuwan A. Fever of unknown origin: a review of 25 cases in Ramathibodi Hospital. J Med Assoc Thai. 1981; 64:155-158. 